\title{
Research on Slope Model Reconstruction Based on Mobile Terminal Monocular Vision
}

\author{
Li Jishan ${ }^{1, ~ *, ~ S h i ~ X i n g x i ~}{ }^{1}$, Wang Xianghong ${ }^{2}$ \\ ${ }^{1}$ School of Science, Nanjing University of Science and Technology, Nanjing, China \\ ${ }^{2}$ China Coal Chang, Jiang Foundation Construction Corporation, Nanjing, China
}

\section{Email address:}

2684073131@qq.com (Li Jishan), xingxishi@163.com (Shi Xingxi)

${ }^{*}$ Corresponding author

\section{To cite this article:}

Li Jishan, Shi Xingxi, Wang Xianghong. Research on Slope Model Reconstruction Based on Mobile Terminal Monocular Vision. Science Discovery. Vol. 9, No. 1, 2021, pp. 1-6. doi: 10.11648/j.sd.20210901.11

Received: December 2, 2020; Accepted: January 8, 2021; Published: January 18, 2021

\begin{abstract}
In this paper, a method of slope surface model reconstruction based on smart phone is proposed for the problems of point layout limitation, expensive measuring equipment and easy operation of monitoring personnel. By explaining the principle and steps of the SfM-MVS algorithm, the slope surface model is reconstructed based on the slope image taken by smart phone. In this paper, the principle of partial reconstruction is described, which involves the principle of polar geometry and projection error, and the corresponding description of motion recovery structure algorithm and dense reconstruction algorithm steps. The concrete steps of slope 3D reconstruction are as follows: first, the mobile phone camera is calibrated by Zhang Zhengyou camera and the slope image is enhanced for subsequent processing. Finally, the SfM-MVS algorithm is used for sparse reconstruction and dense reconstruction to obtain point cloud data, and the slope surface model is reconstructed by triangulation and texture mapping. The slope surface model obtained by smartphone image lays the foundation of slope shape change monitoring and coordinate calculation, and has the characteristics of perfect slope overall information. The model reconstruction based on mobile terminal image acquisition can reduce the equipment cost of slope monitoring and the maneuverability of monitoring personnel, and has certain application value.
\end{abstract}

Keywords: Slope Monitoring, 3D Reconstruction, Smartphone, Monocular Vision

\section{基于移动终端单目视觉的边坡模型重建研究}

李吉珊 ${ }^{*}$, 石杏喜 ${ }^{1}$, 王向红 ${ }^{2}$

1南京理工大学理学院, 南京, 中国

${ }^{2}$ 中煤长江基础建设有限公司, 南京, 中国

邮箱

2684073131@qq.com(李吉珊), xingxishi@163.com(石杏喜)

摘要：针对传统边坡监测中面临的点位布置限制、测量设备昂贵、监测人员操作难易度等问题，本文提出一种基于智能手机 的边坡表面模型重建方法。通过阐述SfM-MVS算法具体原理和步骤进行基于智能手机拍摄的边坡影像进行边坡表面模型的重 建。首先对手机相机进行张正友相机标定并对边坡影像进行图像增强以便后续处理, 其次通过SIFT-RANSAC算法进行特征点 匹配并剔除误差匹配, 最后使用SfM-MVS算法进行稀疏重建和稠密重建获取点云数据, 并使用三角网格剖分和纹理映射完成 边坡表面模型的重建。通过智能手机影像获取的边坡表面模型奠定了边坡形态变化监测和坐标测算的基础, 具有边坡整体信 息完善的特点。基于移动终端获取影像的模型重建能够减少边坡监测的设备成本和监测人员操作性, 具有一定应用价值。 
关键词: 边坡监测, 三维重建, 智能手机, 单目视觉

\section{1. 引言}

我国近年来边坡失稳导致的人民生命财产安全损失 对经济建设以及国计民生都产生了影响, 边坡的规模随着 国内建筑、道桥、矿山等建设工程的持续增加而愈加增长。 因此, 在边坡工程中通过监测进行边坡稳定性评价对安全 生产具有重要意义。

传统边坡监测是以水准仪、全站仪、GNSS、GPS、 雷达等[1-3]方法获取数据信息, 同时由于边坡种类繁多、 监测点布置的局限性、监测设备贵重等原因, 传统监测方 法无法全面反映边坡整体状况。随着计算机视觉、影像匹 配算法、立体视觉重建等技术的不断更新, 近景摄影测量 [4]和无人机倾斜摄影测量 $[5,6]$ 已经能够被用于边坡监测 领域, 但量测相机、非量测相机[7]以及摄测无人机都存在 操作和价格上的限制, 而高像素智能移动终端的普及则将 基于立体视觉三维重建的边坡监测变为可能 [8-9]。本研究 利用智能手机对边坡进行多视角拍摄, 结合影像匹配、 SfM-MVS算法以及三角网格剖分和纹理映射, 实现边坡 的单目多视角三维重建[10], 为边坡稳定性评价奠定基础。

\section{SfM-MVS算法重建流程}

\subsection{SfM算法}

机器视觉中的运动恢复结构 (Structure from Motion)[11]可以用移动相机获取不同视角影像来恢复稀 疏点云, 而多视图立体视觉(Multi-View Stereo)算法[12]则 可以将稀疏点云拓展到稠密点云, 它们能够不依赖地面地 面控制点等先验条件直接从二维影像生成三维点云模型, 具有较高灵活性。 $\mathrm{SfM}$ 的基本原理是以单个移动相机拍摄 的多视角图像序列为输入, 依据图像集中重复区域的午余 信息, 提取同名点的特征匹配关系, 通过求得相机的位姿 及参数计算同名点间对极几何关系来恢复三维场景。SfM 算法本质是一个估计相机参数及点空间位置的问题。

目前主流的SfM算法是增量式SfM, 其具有较高鲁棒 性, 重建场景精度高。增量式SFM重建过程中使用 RANSAC不断过滤外点从而可以不断优化场景结构。因此 增量式SfM契合边坡工程的要求 [13-14]。其算法流程如下:

(1) 对应点搜索

图像匹配中主要关注点在于特征点和特征描述子。特 征点部分包含方向、尺度信息。描述子通常作为向量表示, 用其描述关键点周围像素的信息。在任意一幅图像 $I_{i}$ 中, 第 $N_{F_{i}}$ 个像素点 $x_{j}$ 有特征点:

$$
F_{i}=\left\{\left(x_{j}, f_{j}\right) \mid j=1, \cdots N_{F_{i}}\right\}, \quad x_{j} \in R^{2}
$$

$f_{j}$ 表示第 $j$ 个像素点的描述子, $x_{j}$ 表示第 $N_{F_{i}}$ 个像素 点的位置。在匹配过程中通常需要考虑尺度、光照、视角、
仿射等因素, 来确保两幅含重叠区域的图像中同名特征点 可用相同描述子表达，且保证描述子的区分。采用在视角 变化、仿射变换等方面具有鲁棒性的SIFT算法更适合边坡 三维重建。

特征匹配利用上述已求图像特征 $F_{i}$ 的描述子实现。由 相邻图像 $I_{a} 、 I_{b}$ 中每一特征点描述子的对比, 按相似度最 高视为匹配对从而得到图像匹配对:

$$
C=\left\{\left\{I_{a}, I_{b}\right\} \mid I_{a}, I_{b} \in I, a<b\right\}
$$

以上述方法得到的匹配对因仅依据特征点外观特征 判断而存在大量误匹配, 故采用几何约束来验证匹配, 不 同图像间的相同点存在的映射关系可用单应性矩阵 $H$ 来表 示。图5对极几何中基础矩阵 $F$ 反映点 $p$ 在立体像对中像点 极线映射关系, 即对极几何代数表示。本质矩阵 $E$ 反映点 $p$ 在立体像对中获取两张图像的相机位姿之间的关联, 其不 涉及相机内参。

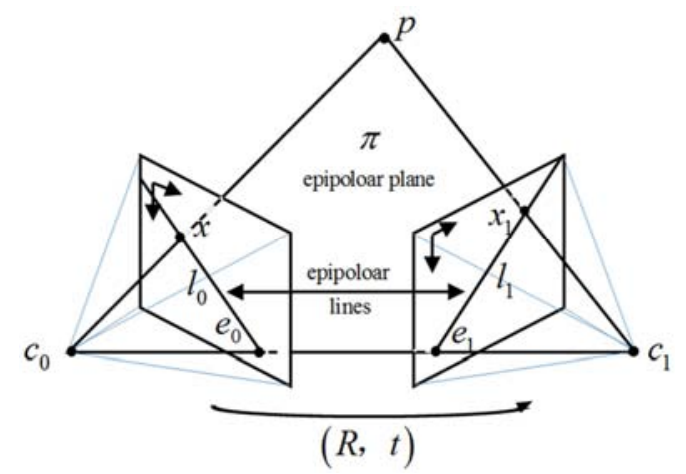

图1 两视图对极几何。

图1中 $p 、 x 、 x_{1} 、 c_{1} 、 c_{2}$ 五点共面, 形成对极平面 $\pi$ 。 $l_{0}$ 、 $l_{1}$ 表示空间点 $p$ 在两幅图中的对极线, $e_{0} 、 e_{1}$ 为对极点。 $x$ 反投影形成的投影射线 $c_{0} p$ 在第二幅图的投影为直线 $l_{1}$, 且 $x_{1}$ 在此直线上。用以下公式完成匹配验证, $p_{0}$ 和 $p_{1}$ 表示两 图像点分别对应的归一化平面图像坐标, $K$ 表示相机内参 数。

$$
\left\{\begin{array}{c}
x_{1}=H x_{0} \\
p_{1}^{T} E p_{0}=0 x_{1}^{T} \\
F x=0, F=K^{-T} E K^{-1}
\end{array}\right.
$$

为避免求解基础矩阵 $F$ 受到噪声干扰, 在获取初始匹 配关系后选用RANSAC算法进行迭代优化, 从而得到满足 对极几何一致性的正确匹配点对。RANSAC选择出可以估 计模型的最小数据集, 然后计算符合该变换（达到预定阈 值）的其他样本，基于契合样本即内点的数量判断继续进 行RANSAC迭代或者停止, 从而消去噪声干扰。

(2) 运动恢复结构 
对应点搜索完毕之后, 通过对极几何估计初始像对初 始化位姿变换, 对两幅图像对应点三角化恢复坐标, 最后 使用光束平差法 (BA) 对参数结果迭代优化。

初始化时选择图像清晰、重复度不低于 $50 \%$ 且相机中 心距离足够远的两幅图像作为初始像对。由 $2 \mathrm{D}-2 \mathrm{D}$ 对应关 系用对极几何(相对定向)估计初始化像对的位姿变换, 利 用本质矩阵 $E$ 可求解相机位姿转换:

$$
E=\left[t_{x}\right] R
$$

本质矩阵 $E$ 可由归一化平面 5 点坐标求得, 相机的旋转 矩阵 $R$ 以及平移向量 $t$ 由本质矩阵进行 $\mathrm{SVD}$ 奇异值分解求 出。

相机位姿关系恢复后, 可以利用三角定位对图像间匹 配到的特征点进行三维信息恢复。通过初始像对中求得的 旋转矩阵 $R$ 以及平移向量 $t$, 可知特征点归一化坐标 $x$ 和 $x_{1}$ 满足公式:

$$
x s=s_{2} R x_{2}+t
$$

完成上式的求解可得深度信息 $s$ 和 $s_{1}$ 的值。

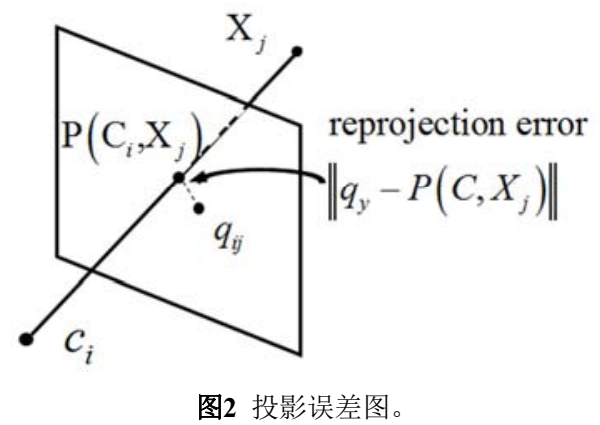

投影误差是投影点和图像中的真实点间的距离, 即图 2 中的箭头表示。所以需要使用光束法平差 (Bundle Adjustment, BA)对初始模型做联合优化, 对手机相机的 $n$ 个位姿和影像的 $m$ 个特征点应满足重投影误差的优化目标 函数:

$$
g(C, X)=\sum_{r=1}^{n} \sum_{j=1}^{m} \omega_{y}\left\|q_{y}-P\left(C, X_{j}\right)\right\|^{2}
$$

根据BA之后的重投影误差统计进行粗差滤除, 滤除 部分平差后重投影误差过大的点或在平差过程中, 根据选 权迭代思想给这些重投影误差过大的点赋比较小的权重。 至此得到两视图稀疏点云, 即通过 $\mathrm{SfM}$ 算法获得初始化模 型。

(3) 增量式重建

初始化过程经过两视图相机位姿估计和场景点三维 信息恢复后, 从初始像对出发, 加入能获取最多数量特征 轨迹的图像进行匹配, 使用透视N点法 (PnP) [15]求解获 取新图像位姿进而得到新点云。由世界坐标系下的现实 $3 \mathrm{D}$ 点 $P_{i}$ 和该点在相机坐标系下的图像 $2 \mathrm{D}$ 点 $v_{i}$ 之间的对应关系 得到世界坐标系和相机坐标系之间的转换公式:

$$
\lambda_{i} v_{i}=R P_{i}+t
$$

其中 $\lambda_{i}$ 为 $v_{i}$ 点深度, $v_{i}$ 表示图像像素坐标。

$\mathrm{PnP}$ 算法根据新增图像中 $\mathrm{n}$ 个点与已经解算完毕的 $\mathrm{n}$ 个点 之间的对应关系, 可以反算出新增图像的旋转矩阵 $R$ 以及平移 向量 $t$ 并以此获得新的相机位姿, 进一步根据图像中的匹配点 和其对应的轨迹关系进行三角化测量, 计算出更多三维点。

由于 $\mathrm{PnP}$ 计算位姿和三角化存在噪声干扰, 估计结果 的偏差往往会随着增量式的过程逐渐增大, 因此在重复新 增图像进行三维点添加的过程中需要不断地利用BA对求 解结果进行优化。在所有步骤结束后会再对整个结果进行 一次光束法平差优化。

\subsection{MVS算法}

稠密重建MVS是一种基于多视角的匹配重建算法, 其 输入一般采用 $\mathrm{SfM}$ 相机网络, 最终输出的为密集三维点云 并附带目标相应位置RGB信息。一般先使用CMVS算法[16] 将图像通过聚簇分类以优化匹配效率, 然后经过 PMVS[17-18]算法的匹配、扩散、过滤几个步骤迭代达成 密集匹配的目的。

散列图像聚簇或CMVS算法要求聚簇满足无攵余图 像、簇小且能完成重建、簇重建结果可保持影像完整。其 主要步骤为: (1) $\mathrm{SfM}$ 篮选: 重复用各领域位置均值表 示SfM局部领域特征点信息, 从而降低SfM点集数目并获 取输出点集; (2) 影像获取: 以分辨率大小排序搜索, 剔除分辨率不足和不契合覆盖约束的图像; (3) 聚簇分 类: 忽略覆盖约束并以图像大小为约束, 采用标准图像分 割算法进行影像分割并继续分割不满足图像大小约束的 簇。（4）增添影像：为各个未加入的特征点构建图像簇 并依据SfM效率最大将图像加入簇的行为。重复最后两步 直至获得符合覆盖约束以及影像大小约束的聚簇结果。

基于特征点扩散的PMVS算法具有点云精度高和分布 均匀等优点, 其主要步骤为: (1) 匹配: 用 $\mathrm{SfM}$ 重建生 成的稀疏点云生成一种尺度小并近似正切于重建对象表 面的矩形作为稀疏面片; (2) 扩散: 从稀疏种子点拓展 到密集点云, 得到更加稠密的面片序列进行重构; (3) 过滤: 依据连续可视性剔除不在实际表面的内点和外点。 通过第二步和第三步的多次重复迭代生成稠密点云。

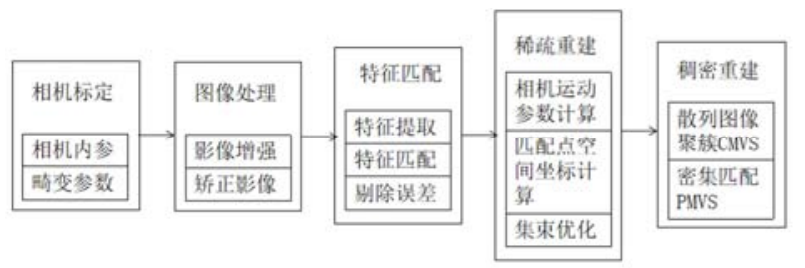

图3 SfM-MVS重建流程。

\section{3. 边坡三维建模及分析}

\section{1. 相机参数获取以及边坡影像处理}

对手机相机采用机器视觉中广泛使用的张正友标定 法[19]进行标定, 由于手机相机镜头不存在大畸变, 同时 
标定实验便于实现, 故选用此方法。本次标定采用电脑屏 幕1:1显示A4纸大小棋盘格, 避免了打印和粘贴棋盘格而 产生的纸面形变。本次笔者常用的华为nova2s手机进行标 定。标定结果为:

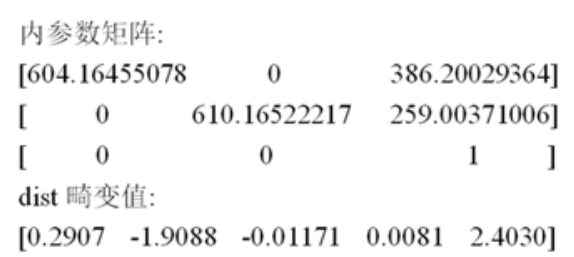

得出相机内参和畸变参数后查看角点标记图和相机 畸变校正效果, 如图5。
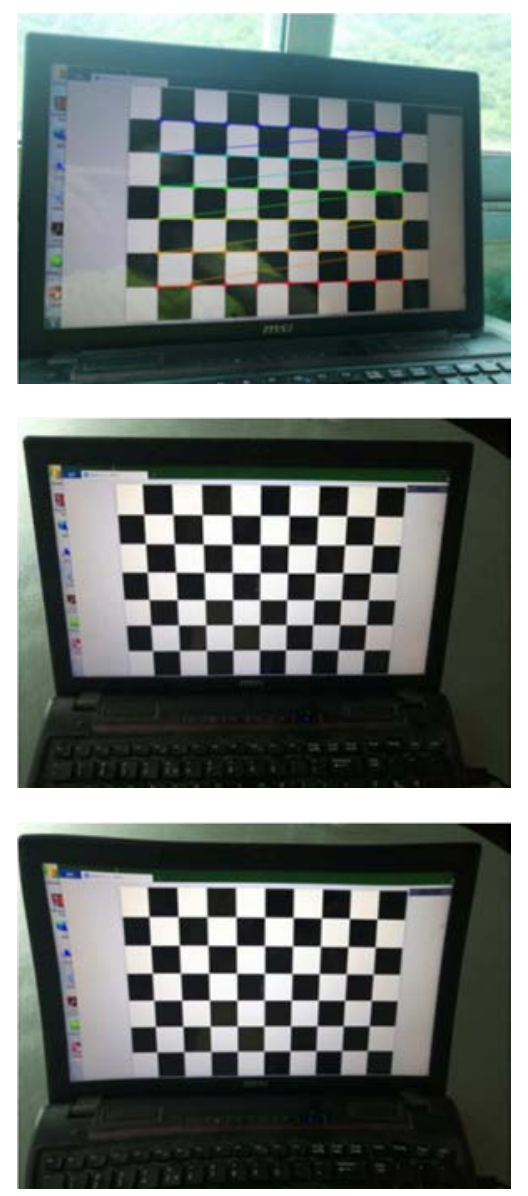

图4 角点标记和畸变校准图。

选取校内理学院门口新建工程边坡, 使用智能手机对 同一部位进行多角度重复拍照, 获取部分影像如下:

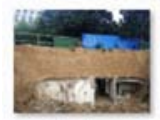

001.jpg

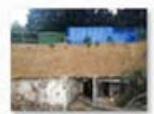

006.jpg

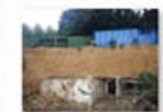

002.jpg

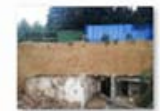

007.jpg

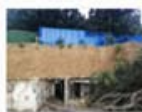

003.jpg

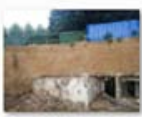

008.jpg

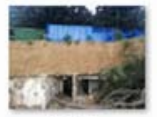

004.jpg

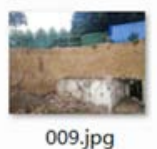

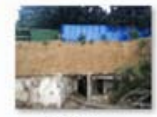

005.jpg

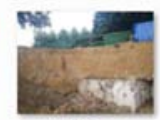

010.jpg
图5 部分边坡图像。
此外，为使相片更适合计算机处理，选择进行图像增 强来突出其有用信息。

\section{2. 特征匹配}

SIFT特征具有视角改变、仿射变换以及噪声条件下的 稳定性, 其基本原理是使用高斯卷积核来获得图像不同的 尺度空间，在多尺度空间下寻找极值点。SURF和SIFT算 子的特性符合边坡三维重构计算其表面位移和变形的要 求 [20], 同时SIFT算子相较于SURF尺度、旋转以及旋转变 化上有明显优势, 故SIFT算子更适合边坡影像的特征检测。 提取效果如下:
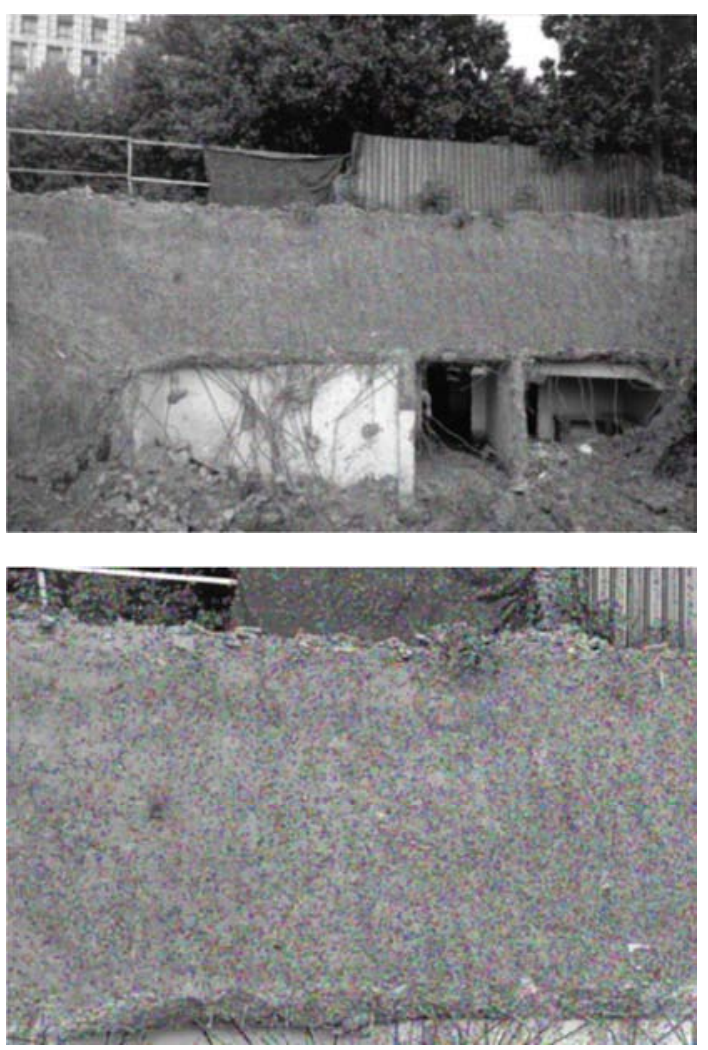

图6 SIFT算子特征提取。

在使用SIFT算法特征进行匹配时容易因环境因素产 生较多误匹配即产生噪声, 选择采用RANSAC(Random Sample Consensus)算法来消除误匹配。随机抽样一致性算 法[21]通过随机抽取观测数据来估计模型的各个参数。对 于一组包含噪声的数据集, RANSAC算法通过比选来找出 最优适配模型。利用RANSAC算法消除SIFT误匹配点流程 如下: (1) 随机选取样本集中的一组 (4个) 匹配点; (2) 计算变换矩阵 $\mathrm{M} ;$ （3）返回变换矩阵 $\mathrm{M}$ 与矩阵内符合误 差度量函数的一致集里的元素个数; (4) 更新满足当前 元素数为最大的最优一致集; (5) 更新此时的错误概率 $p$ 。 在 $p$ 满足最小错误概率条件前进行以上步骤迭代。直接进 行匹配与RANSAC消除误匹配对比如下: 

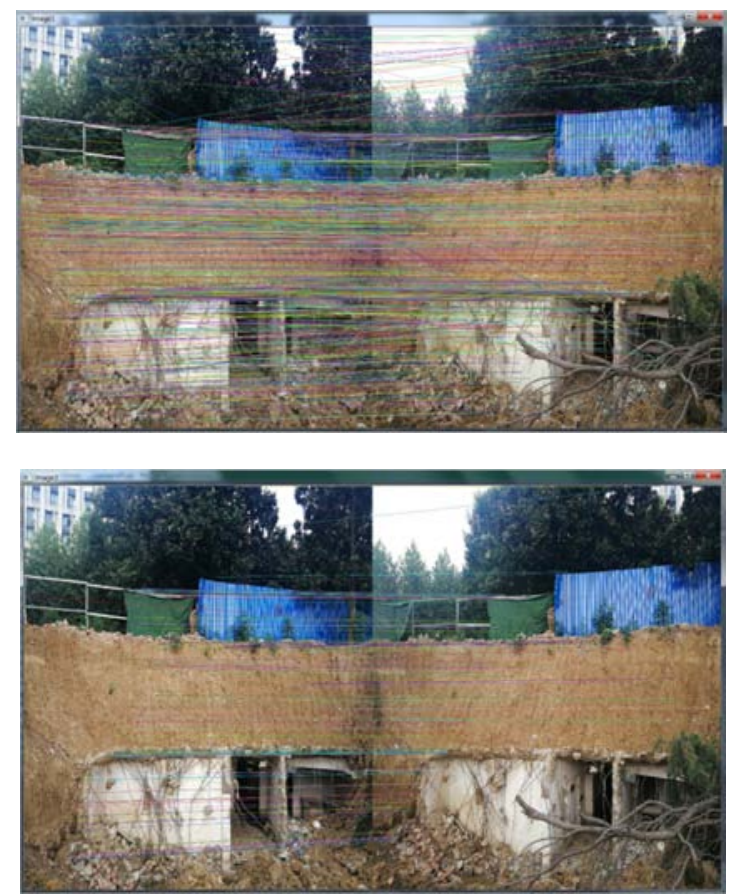

图7 SIFT匹配与SIFT-RANSAC匹配对比。

\section{3. 稀疏重构与密集重构}

稀疏重构即使用匹配好的特征点坐标进行三维重构, 通过点云可视化函数获取各点在三维空间中的位置并通 过窗口展示和点云文件输出, 增量式SfM重建生成稀疏点 云效果如下:

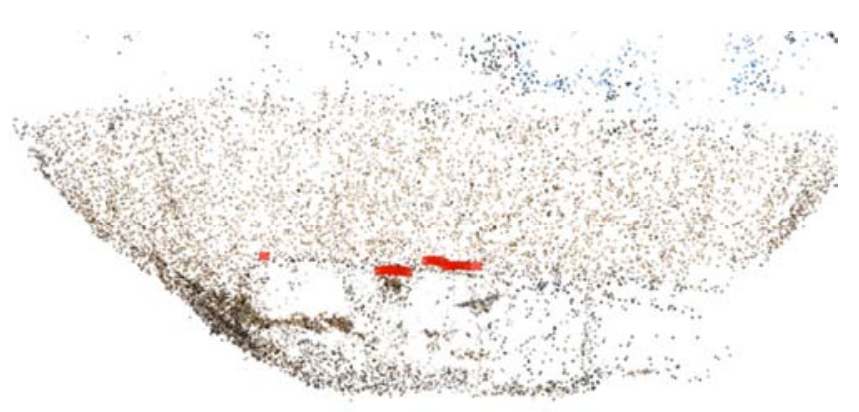

图8 SfM重建稀疏点云。

相较于特征明显形状规则的物体, 稀疏重建对于边坡 这类不规则物体缺少细节特征, 同时点云过于稀疏也会影 像后续建立表面模型的精度，从而失去边坡稳定性评价的 意义, 因此采用基于特征点扩散的MVS密集重建能够很好 保证点云精度和规模。稠密重建效果如下:

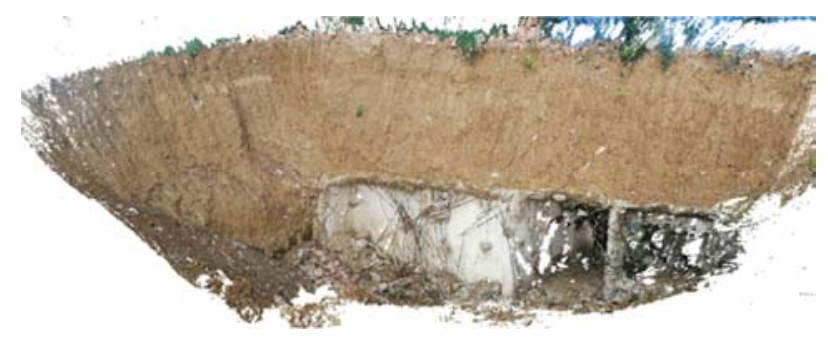

图9 密集重建效果图。

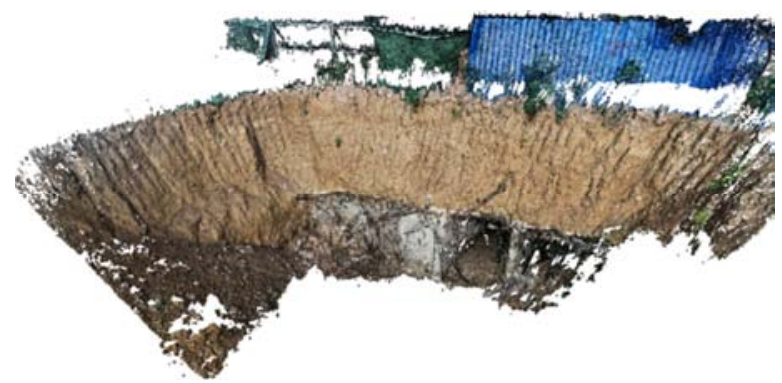

图10 稠密重建点云（特征点尺度增大）。

密集重建后的点云依旧是离散的, 点云数据无法有效 反映边坡的地形结构。选择三角剖分通过生成表面模型的 方法来为后续计算提供基础，Delaunay三角剖分算法[22] 因其具备空圆特性和最大化最小角特性而广泛应用于测 绘学等。Delaunay三角网 (D-TIN) 剖分过程中不会生成 过于尖锐的三角形, 能够较为真实的反映边坡表面形态。

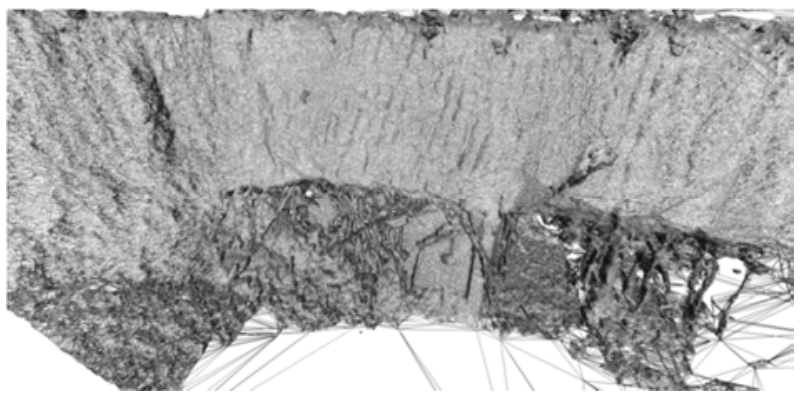

图11 Delaunay三角剖分效果。

三角剖分之后通过纹理映射来添加现实场景中的边 坡纹理, 纹理映射原理是将纹理空间中的纹理像素映射到 屏幕空间中的像素[23]。其主要步骤就是将二维纹理平面 映射到三角剖分后的三维网格上。纹理映射反映边坡色彩 细节便于对照边坡模型变化, 同时能够为后续根据输入已 知监测点大地坐标来推算待求监测点坐标提供有利条件。 三角网格模型纹理映射效果如下:

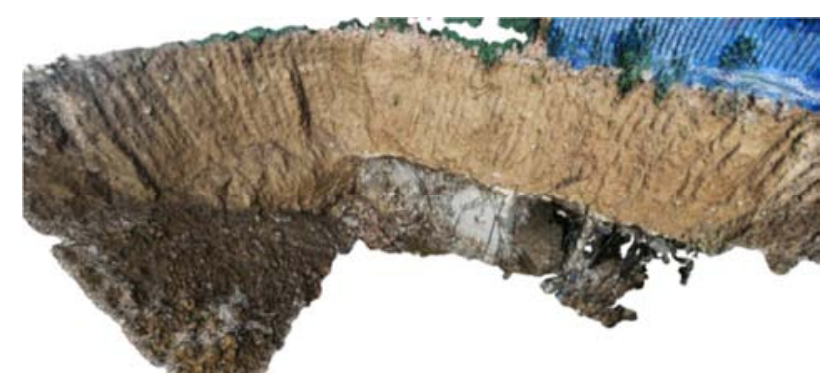

图12 纹理映射效果图。

\section{4. 结果分析}

由结果图可以看出, 基于手机相机的边坡三维重建能 够直观地表现边坡地形特征，在后续计算边坡位移时可以 选择边坡表面模型对比的计算方式，对在进行三角剖分时 生成的D-TIN亦可用于DEM分析。边坡位移计算可在不同 施工时间段分别进行边坡影像拍摄并三维重建, 再将两次 
重建模型置于同一坐标下进行相减得出不同部位表面变 化以及各向位移如 $\Delta \mathrm{X} 、 \Delta \mathrm{Y} 、 \Delta \mathrm{Z}$ 从而达到监测目的。

\section{4. 结论}

针对传统近景摄影测量在边坡工程监测中技术要求 高、监测设备笨重昂贵、无法及时监测边坡整体状况以及 监测人员操作耗时等问题, 结合智能手机的普及以及手机 相机拍摄分辨率提高的现状，提出了基于智能手机单目视 觉三维重建在边坡监测中应用的方法。该方法将相机进行 张正友标定并对拍摄的边坡影像进行图像增强处理, 通过 SfM-MVS算法获取边坡影像特征点的点云数据再使用 Delaunay三角剖分进行重建并纹理映射获取具有纹理特 征的边坡表面模型。此表面模型能够为后续坐标推算以及 边坡形态变化监测提供有利条件, 不同时间段的重建模型 对比能够得到边坡不同部位表面变化和各向位移。同时该 方法由于无需量测相机和传统非量测相机, 只需监测人员 使用智能手机进行图像采集, 较大的节约了仪器采购成本。 而监测人员的操作流程亦不复杂, 减少了监测人员的操作 难易度并提高了监测效率。重建的表面模型相比于传统点 位监测具有监测对象更全面的优点, 能够较好地反映边坡 的整体信息。通过将点云数据进行DEM分析能够获取更多 信息, 而配合少量人工监测点位的布置以及坐标测量可以 获取更为精确的尺度信息, 后续将进行增设已知点从而推 算未知点的研究, 通过坐标推算与传统测量方法进行比照 来分析精度和边坡稳定性评价。

\section{参考文献}

[1] 崔春晓,朱自强,杨光轩,等.基于GNSS技术的排土场边坡监 测及稳定性研究 [J].中国矿业,2020,029(003):94-99。

[2] 张爱军.GPS技术在边坡监测中的应用研究[J]. 商品与质量, 2016,000(048):286-287。

[3] 间国斌,陶志刚,孙光林,等.边坡雷达在矿区边坡监测区域的 应用分析 [J].工业安全与环保,2015,000(010):57-60。

[4] 谢冬冬,杨德宏. 近景摄影技术在露天矿山边坡变形监测中 的应用[J].价值工程,2016(21):123-124,共2页。

[5] 桂林,陈昌黎,谢飞,等.倾斜摄影测量在红石岩堰塞体高边坡 治理工作中的应用 $[C] / 2018$ 年西南5省,市,区第二次岩石力 学与工程学术大会论文集(下册:试验与研究监测与测量综 合性技术).2018。

[6] 李竹有,杨国华,孙洪稳,等.无人机非接触式高边坡表面位移 监测方法[J].云南水力发电,2019,035(0z2):30-35。
[7] 唐旭.近景摄影测量在边坡监测中的应用研究[D].2016。

[8] 陈思园,宋展,尹业安.基于智能手机的三维重建方法[J].计算 机工程,2019,45(03):231-237+242。

[9] 张宁,王竞雪.基于智能手机立体影像的稠密三维重建 [J].遥 感信息,2020,035(002):87-93。

[10] 王冬.基于单目视觉的三维重建技术研究[D].2016.

[11] 韩超.基于无人机航拍图像的三维重建 SfM算法研究 [D]. 2019.

[12] 李兆歆.多视角立体三维重建方法研究[D].

[13] Peppa M V, Mills J P, Moore P, et al. Automated co-registration and calibration in SfM photogrammetry for landslide change detection: Automated SfM co-registration for landslide change detection [J]. Earth Surface Processes and Landforms, 2018, 44.

[14] Esposito G, Salvini R, Matano F, et al. Multitemporal monitoring of a coastal landslide through SfM - derived point cloud comparison [J]. The Photogrammetric Record, 2017, 32 (160): 459-479.

[15] 童张海.单目视觉空间目标位姿测量算法研究[D].哈尔滨工 业大学。

[16] 何谦.基于单目视觉的环境稀疏重建与稠密重建算法研究 [D]。

[17] Shuangting W, Kunkun C, Xiaolong L. An improved PMVS algorithm based on multi-view oblique images [J]. Journal of Henan Polytechnic University(Natural ence), 2015.

[18] Wang J A, Ma H T, Wang C M, et al. Fast 3D reconstruction method based on UAV photography [J]. Etri Journal, 2018, 40 (6): 788-793.

[19] 迟德霞,王洋, 宁立群, 等.张正友法的摄像机标定试验 [J].中 国农机化学报,2015, 36(002):287-289。

[20] Luo J, Oubong G. A Comparison of SIFT, PCA-SIFT and SURF [J]. International Journal of Image Processing, 2009.

[21] Chum, Ondřej, Matas, Jiří. Optimal Randomized RANSAC[J]. IEEE Transactions on Pattern Analysis \& Machine Intelligence, 2008, 30 (8): 1472-1482.

[22] CHEN Tingwang, WANG Qing, 陈庭旺,等. Triangulation algorithm based on point clouds reconstructed by SfM一种基 于 $\mathrm{SfM}$ 重建点云的三角网格化算法*[J]. 计算机应用研 究,2011, 28(2):794-796。

[23] Schuster K, Trettner P, Schmitz P, et al. A Three-Level Approach to Texture Mapping and Synthesis on 3D Surfaces [J]. Proceedings of the ACM on Computer Graphics and Interactive Techniques, 2020. 